\title{
Tissue Immunofluorescence Confirmation of CNS Autoantibodies Identified by Immunoblot or Cell- Based Assay
}

\section{Rania W. Abelhosn}

Quest Diagnostics

\section{Laura Montana}

Quest Diagnostics

Jonnielyn G. Rivera

Quest Diagnostics Nichols Institute San Juan Capistrano: Quest Diagnostics Nichols Institute Farnoosh Haji-Sheikhi

Quest Diagnostics Nichols Institute San Juan Capistrano: Quest Diagnostics Nichols Institute Joanne H. Diao

Quest Diagnostics Nichols Institute San Juan Capistrano: Quest Diagnostics Nichols Institute Harley H. Tran

Quest Diagnostics Nichols Institute San Juan Capistrano: Quest Diagnostics Nichols Institute

\section{Michael Levy}

Massachusetts General Hospital

Anthony A. Horner ( $\square$ anthony.a.horner@questdiagnostics.com )

Quest Diagnostics Nichols Institute

\section{Short report}

Keywords: Paraneoplastic syndrome, CNS autoantibody detection, Immunofluorescence assay, Immunoblot assay, Cell based assay

Posted Date: December 15th, 2020

DOI: https://doi.org/10.21203/rs.3.rs-126755/v1

License: (c) (i) This work is licensed under a Creative Commons Attribution 4.0 International License. Read Full License 


\section{Abstract}

Background: While many immunologic targets of autoimmune CNS disease have been identified, autoantibodies to each, are detected only rarely. Therefore, serum is often screened by immunofluorescence assay (IFA) with whole brain tissue before reflexing positive samples to more specific assays for confirmation and identification. The purpose of this study was to compare the sensitivity of immunofluorescence positive staining of whole brain tissue to immunoblot assays (IBAs) and cell-based immunofluorescence assays (CBAs) for the initial detection of autoantibodies associated with paraneoplastic syndrome (PNS) and other autoimmune diseases of the CNS.

Methods: All comprehensive paraneoplastic neurological syndrome panel results released from our laboratory over a two-year period (2017-2019) were reviewed. This panel was specifically chosen for these retrospective analyses because of the comprehensive PNS panel's design; a reflex testing algorithm is not used and a tissue IFAs, IBAs, and CBAs, are run in parallel and independently on all serum samples.

Results: For autoantibodies to intracellular targets, tissue IFA results were positive for 47 of 56 (84\%) samples that were positive by IBA. In contrast, for autoantibodies to membrane-imbedded antigenic targets, tissue IFA results were positive for only 5 of 14 (35.7\%) samples that were positive by CBA.

Conclusions: These retrospective analyses suggest that tissue IFAs of serum are more than twice as likely to confirm the presence of IBA identified autoantibodies to intracellular proteins than CBA identified autoantibodies to membrane imbedded protein targets. Moreover, these findings have important implications for the use of tissue IFA screening for the initial detection of CNS autoantibodies.

\section{Introduction}

Over the last couple of decades, autoimmune diseases of the CNS have become a major focus of investigation within the field of neurology. Armed with an expanding arsenal of commercially available diagnostic tests, the medical community has come to appreciate that neuronal autoantibodies may directly cause or serve as biomarkers for a far wider range of CNS diseases than previously realized $(1,2)$. These include demyelinating diseases, encephalopathy, dementia, epilepsy, and ataxia. Neuronal autoantibody production is often associated with malignancy but not always.

Current thinking is that malignant cells have the potential to both express and break tolerance to neuronal antigens, thereby eliciting an autoimmune reaction within non-malignant tissues, including the brain, optic nerves, spinal cord, peripheral nerves, neuromuscular junction, and muscle (3). This likely explains why most CNS specific autoantibodies are more readily detected in serum than in cerebral spinal fluid.

Interestingly, most malignancy associated CNS autoantibodies target intracellular proteins. These autoantibodies are probably not directly pathogenic, but instead serve as surrogate markers of underlying T-cell mediated CNS pathology (3). Neuronal cell surface proteins associated with neurotransmitter receptors and ion channels represent a separate class of antigenic targets whose association with cancer 
is much weaker. Moreover, these autoantibodies are thought to be directly pathogenic and contribute to neurological symptoms and disease (1-3). These views are supported by several observations. For example, CNS diseases associated with intracellular autoantibodies tend to be more responsive to therapies that broadly target cellular immunity, such as high dose corticosteroids, while clinical responses are often partial rather than complete $(1,2)$. In contrast, diseases associated with autoantibodies to neuronal cell surface proteins are more responsive to plasmapheresis and intravenous immunoglobulin therapy, and neurological recovery is often rapid, complete, and sustained $(1,2)$.

While many intracellular and membrane targets of CNS disease have been identified, autoantibodies to each are detected only rarely $(4,5)$, and there are likely additional antigenic targets that have yet to be identified $(4,5)$. Therefore, reference laboratories often use immunofluorescence assays (IFAs) that include cerebellum, hippocampus, and potentially other tissues for the initial detection of CNS autoantibodies. Confirmatory testing is then done with assays designed to identify autoantibodies to individual target proteins. Of the techniques available, immunoblot assays (IBAs) are considered preferable for detecting autoantibodies to most intracellular targets, while detection of autoantibodies to membrane embedded proteins is generally better achieved with cell-based assays (CBAs) that utilize HEK293 cells transfected to express the protein of interest in its native conformation $(4,5)$. Tissue IFAs have historically been considered very sensitive for the detection of autoantibodies. However, little is known about the sensitivity of tissue IFAs relative to IBAs and CBAs for the initial detection of autoantibodies to intracellular and membrane embedded proteins, respectively. To address this issue, we conducted retrospective analyses of tissue IFA, IBA, and CBA results from patient serum samples sent to our laboratory for concurrent CNS autoantibody testing by all three methods.

\section{Methods}

\section{Study Design}

To determine the frequency with which IBA and CBA identified CNS autoantibodies are also detected by tissue IFA, we reviewed all comprehensive paraneoplastic neurological syndrome (PNS) panel results released from our laboratory over a two-year period (2017-2019). Results from this panel were selected because of the comprehensive PNS panel's design; a reflex testing algorithm is not used and tissue IFA, IBAs, and CBAs were run in parallel on each serum sample. Therefore, each tissue IFA result was obtained by lab technicians unbiased by prior knowledge of the IBA and CBA results. Only IBA and CBA identified autoantibodies and their corresponding tissue IFA results were considered in these analyses.

\section{Laboratory Techniques}

Immunoblot assays were conducted with the Euroimmun (Lubeck Germany) Euroline PNS 12 antigen profile (DL 1111-1601-7 G), which includes individual immunoblots for Hu, Yo, Ri, CV2/collapsin response mediator protein 5 (CRMP5), Ma2, anti-glial nuclear antibody (AGNA)/SOX1, Zic4, glutamic acid decarboxylase 65 (GAD65), amphiphysin, delta/notch-like epidermal growth factor-related receptor (DNER)/Tr, titin, and recoverin. Assays were conducted with the Euroblot One system, following 
manufacturer recommendations. Results were reported in signal intensity $(\mathrm{SI})$ units and those $\geq 11 \mathrm{SI}$ units were considered positive. Cell-based assays were conducted with the Euroimmun encephalitis mosaic panel 1 (FA 112d-1), which includes HEK293 cells transfected with a-amino-3-hydroxy-5-methyl-4isoxazolepropionic acid receptor 1 and 2 (AMPAR1, AMPAR2), N-methyl-D-aspartate receptor (NMDAR1), contactin-associated protein 2 (CASPR2), gamma-aminobutyric acid B receptor (GABAbR) and leucinerich glioma-inactivated protein 1 (LGI-1) according to the manufacturer's instructions. Each CBA was initially run at a 1:10 dilution with serial 1:2 dilutions until endpoint. Slides were interpreted with the EuroPattern imaging system. Tissue IFAs were conducted with custom-designed Euroimmun slides that contain 9 tissues, including monkey cerebellum and rat hippocampus. Sera were processed according to manufacturer guidelines and images were interpreted with the EuroPattern imaging system at a 1:40 dilution. Although titin and recoverin antigens are included on the immunoblot strips used for these studies, tissues appropriate for autoantibody detection were not included on the tissue IFA slide, and therefore, these analytes were not included in the analyses.

\section{Statistics}

The non-parametric Wilcoxon rank sum test was used to determine if the signal strength of IBA and CBA detected (SI and titer, respectively) CNS autoantibodies influenced the likelihood that they would also be detected by tissue IFA. A chi-square test was used to compare the relative sensitivity of the tissue IFA for the detection of IBA and CBA identified CNS autoantibodies. $P$-values $\leq 0.05$ were considered statistically significant. Statistical analyses were conducted using $\mathrm{R}$ version 3.6.3, R Foundation for Statistical Computing (https://www.R-project.org).

\section{Results}

Fifty-four serum samples with positive IBA results were identified that met study criteria. Two of the samples had autoantibodies to two target CNS proteins, while the rest had a single IBA detected CNS autoantibody. Of these 56 IBA identified CNS autoantibodies, 47 (84\%) were also positive by tissue IFA (Table 1). The mean IBA signal intensities (SIs) of tissue IFA positive and negative samples were $36.7 \mathrm{SI}$ (S.D. \pm 37.1$)$ and 22.3 (S.D. \pm 6.4$)$, respectively $(P=0.6)$. Although this difference was not statistically significant, it is noteworthy that $78 \%$ (33/42) of IBA detected CNS autoantibodies with a relatively weak signal $(\mathrm{SI}=11-31)$ were confirmed by tissue IFA, while $100 \%(14 / 14)$ of IBA detected CNS autoantibodies with a stronger signal $(\mathrm{SI}=32-175)$ were confirmed. 
Table 1

Tissue IFA detection of IBA identified CNS autoantibodies

\begin{tabular}{|c|c|c|c|c|c|}
\hline \multirow[b]{2}{*}{ Analyte } & \multicolumn{2}{|c|}{ Detected By Tissue IFA } & \multicolumn{2}{|c|}{$\begin{array}{l}\text { Not Detected By } \\
\text { Tissue IFA }\end{array}$} & \multirow{2}{*}{$\begin{array}{l}\text { Assay } \\
\text { Agreement }\end{array}$} \\
\hline & $\mathbf{N}$ & Sample SI (Mean \pm S.D.) & $\mathbf{N}$ & $\begin{array}{l}\text { Sample SI } \\
\text { (Mean } \pm \text { S.D.) }\end{array}$ & \\
\hline $\mathrm{Hu}$ & 3 & $18,48,114(60)$ & 0 & & $\begin{array}{l}3 / 3 \\
(100 \%)\end{array}$ \\
\hline Yo & 8 & $12,14,16,30,33,44,90,96$ (41.9) & 1 & 31 (31) & $\begin{array}{l}8 / 9 \\
(88.9 \%)\end{array}$ \\
\hline Ri & 0 & & 0 & & $0 / 0(N A)$ \\
\hline CV2/CRMP5 & 1 & $17(17)$ & 2 & $14,25(19.5)$ & $\begin{array}{l}1 / 3 \\
(33.3 \%)\end{array}$ \\
\hline Ma2 & 3 & $12,12,50(24.7)$ & 0 & & $\begin{array}{l}3 / 3 \\
(100 \%)\end{array}$ \\
\hline AGNA/SOX1 & 14 & $\begin{array}{l}\begin{array}{l}12,13,14,14,14,15,17,20,20,21,25,25,30,30 \\
(19.3)\end{array}\end{array}$ & 2 & $12,20(16)$ & $\begin{array}{l}14 / 16 \\
(87.5 \%)\end{array}$ \\
\hline Zic4 & 3 & $11,12,16(13)$ & 3 & $17,22,27(22)$ & $3 / 6(50 \%)$ \\
\hline GAD65 & 9 & $14,16,17,21,28,32,111,128,175(60.2)$ & 0 & & $\begin{array}{l}9 / 9 \\
(100 \%)\end{array}$ \\
\hline Amphiphysin & 5 & $12,13,26,60,97(41.6)$ & 1 & $15(15)$ & $\begin{array}{l}5 / 6 \\
(83.3 \%)\end{array}$ \\
\hline Tr/DNER & 1 & $58(58)$ & 0 & & $\begin{array}{l}1 / 1 \\
(100 \%)\end{array}$ \\
\hline Total & 47 & $(36.7 \pm 37.1)$ & 9 & $(20.3 \pm 6.4)^{*}$ & $\begin{array}{l}47 / 56 \\
(84 \%)\end{array}$ \\
\hline
\end{tabular}


Table 2

Tissue IFA detection of CBA identified CNS autoantibodies

Detected By Tissue IFA

Not Detected By Tissue IFA

Assay

Agreement

Analyte N Titer of each sample (Mean $\pm \quad N \quad$ Titer of each sample (Mean \pm

S.D.)

$\begin{array}{llll}\text { AMPAR1 } & 0 & 0 & 1 / 3 \\ & & (33.3 \%)\end{array}$

$(33.3 \%)$

AMPAR2 0

0

$0 / 0(N A)$

NMDAR $111: 160(1: 160)$

$2 \quad 1: 10,1: 80(1: 45)$

$1 / 3$

$(33.3 \%)$

CASPR 2 1:160, 1:160 (1:160)

$4 \quad 1: 10,1: 20,1: 40,1: 80(1: 38)$

$2 / 6$

2

\begin{tabular}{llllll} 
GABAbR & 1 & $1: 80(\mathbf{1 : 8 0})$ & 1 & $1: 20(\mathbf{1 : 2 0})$ & $1 / 2(50 \%)$ \\
\hline LGI-1 & 1 & $1: 20(\mathbf{1 : 2 0 )}$ & 2 & $1: 10,1: 20(\mathbf{1 : 1 5 )}$ & $1 / 3$ \\
& & & & & $(33.3 \%)$
\end{tabular}

Total $\quad 5 \quad(1: 116 \pm 63.9)$

$9 \quad(1: 32.2 \pm 28.6) *$

$5 / 14$

$(36 \%)$

*P-Value $=0.02 ;$ None of the samples were positive for AMPAR1 or AMPAR2

Fourteen serum samples with CBA detected CNS autoantibodies were identified that met study criteria. Each of them was positive for a single autoantibody and none had an additional IBA detected CNS autoantibody. Of these 14 autoantibodies, only 5 were also detected by tissue IFA (35.7\%). The mean CBA titer of tissue IFA confirmed CNS autoantibodies was 1:116 (S.D. $\pm 1: 63.9$ ) compared to 1:32.2 (S.D. \pm 1:28.6; $P=0.02$ ) for tissue IFA unconfirmed CNS autoantibodies. However, the tissue IFA still only detected $57.1 \%$ (4/7) of CBA identified CNS autoantibodies with a titer $\geq 1: 40$ and $14.2 \%(1 / 7)$ of autoantibodies with low titers $(1: 10$ and $1: 20)$. Finally, the tissue IFA was significantly less sensitive in detecting CNS autoantibodies identified by CBA than those identified by IBA ( $35.5 \%$ vs $84 \%$, respectively; $P=0.001)$.

\section{Conclusions}

Many laboratories currently use tissue IFA screening as a predicate method to detect a wide range of CNS autoantibodies in serum. Subsequently, IBAs and CBAs are used to confirm the tissue IFA result and identify the precise antigen specificity of the autoantibody. However, one potential limitation of this approach is that clinically relevant CNS autoantibodies would be missed if not initially detected by tissue IFA. To address this concern, we conducted a retrospective study to determine how often tissue IFA results confirmed the presence of CNS autoantibodies identified by IBA or CBA. The tissue IFA was found to detect $84 \%$ of the CNS autoantibodies identified by IBA but only $34 \%$ of those identified by CBA. In 
consideration of these findings, it is worth considering that the specific antigenic targets of IBAs used for these studies were all intracellular proteins, while CBAs were used exclusively for the detection of autoantibodies to cell surface receptors and other membrane embedded proteins. These considerations strongly suggest that the tissue IFA is more sensitive for the detection of autoantibodies to intracellular proteins than to membrane embedded proteins.

While not available for studies described herein, other investigators have used clinically characterized serum samples to compare the performance of the tissue IFA in detecting CNS autoantibodies initially identified by IBA, and their clinical significance (6). When the clinical histories of 58 patients with an IBA detected and tissue IFA confirmed CNS autoantibody were reviewed, all had a neurological syndrome consistent with the autoantibody identified. Moreover, 50 of the 58 patients also had a malignancy known to be associated with the identified CNS autoantibody. In contrast, IBA detected autoantibodies with a low SI were often not confirmed by tissue IFA, were far less likely to be the cause of the patient's neurological symptoms, and were rarely associated with malignancy (6). Considered in conjunction with our own results, the findings of this study suggest that clinically relevant CNS autoantibodies to intracellular targets are unlikely to be missed by tissue IFA screening of serum, especially if present at higher concentrations, while those that are missed, are far less likely to be of clinical consequence.

Consistent with our findings, other published investigations have concluded that CBAs offer significantly better sensitivity than tissue IFAs for the detection of serum autoantibodies to select membrane embedded proteins, including Aquaporin-4, myelin oligodendrocyte glycoprotein, and NMDAR (7-11). Moreover, unlike IBA detected autoantibodies of low SI, which often are not clinically relevant (6), a vast majority of CNS autoantibodies detected by CBA have clinical relevance (7-11). Considered in this context, findings presented previously suggest that potentially pathogenic CNS autoantibodies with membrane protein specificities, which are readily detected by CBA, are often missed by tissue IFA.

In summary, these findings support the continued use of the tissue IFA as a sensitive screening test for the initial detection of CNS autoantibodies to intracellular target proteins. Moreover, the tissue IFA has clinical utility for the detection of CNS autoantibodies to currently unidentified target proteins that may be of clinical relevance. However, based on retrospective analyses presented in this paper, we strongly recommend that CBAs be included in the initial evaluation of all patients suspected of having autoimmune mediated CNS diseases.

\section{List Of Abbreviations}

PNS - Paraneoplastic Syndrome

IFA - Immunofluorescence assay

CBA - Cell based assay

IBA - Immunoblot assay 


\section{Declarations}

- Ethics approval and consent to participate: Our internal IRB was consulted. These studies did not require their approval

- Consent for publication: Not applicable

- Availability of data and materials: Not applicable

- Competing interests: While studies presented herein did not receive independent research funding. However, all authors, except Michael Levy, are Quest Diagnostics employees.

- Funding: These studies did not receive independent funding

- Authors' contributions: RWA and LM collected data and prepared tables for this paper. LM, JGR, JHD HHT were largely responsible for generating the data presented in this paper. FXHS conducted all pertinent statistical analyses for the paper. $M L$ helped craft the paper and provided critical editorial input. AAH oversaw data collection and statistical analyses. Moreover, AAH wrote a majority of the paper and is primarily responsible for its content

- Acknowledgements: The authors wish to thank the entire Quest diagnostics immunology laboratory operations team for their continued efforts to provide excellent service to the clinicians we serve and for generating the data included in these analyses. The authors also wish to thank Michael Racke, MD, for his critical review of the paper.

\section{References}

1. McKeon A, Pittock SJ. Paraneoplastic encephalomyelopathies: pathology and mechanisms. Acta Neuropathol. 2011;122(4):381-400. doi:10.1007/s00401-011-0876-1

2. Galetta KM, Bhattacharyya S. Multiple Sclerosis and Autoimmune Neurology of the Central Nervous System. Med Clin North Am. 2019;103(2):325-336. doi:10.1016/j.mcna.2018.10.004

3. Bradl M, Lassmann H. Neurologic autoimmunity: mechanisms revealed by animal models. Handb Clin Neurol. 2016;133:121-143. doi:10.1016/B978-0-444-63432-0.00008-6

4. Naides SJ. The role of the laboratory in the expanding field of neuroimmunology: Autoantibodies to neural targets. J Immunol Methods. 2018;463:1-20. doi:10.1016/j.jim.2018.04.003

5. Waters P, Pettingill P, Lang B. Detection methods for neural autoantibodies. Handb Clin Neurol. 2016;133:147-163. doi:10.1016/B978-0-444-63432-0.00009-8

6. Déchelotte B, Muñiz-Castrillo S, Joubert B, et al. Diagnostic yield of commercial immunodots to diagnose paraneoplastic neurologic syndromes. Neurol Neuroimmunol Neuroinflamm. 2020;7(3):e701. Published 2020 Mar 13. doi:10.1212/NXI.0000000000000701

7. Waters PJ, McKeon A, Leite MI, et al. Serologic diagnosis of NMO: a multicenter comparison of aquaporin-4-IgG assays. Neurology. 2012;78(9):665-669. doi:10.1212/WNL.0b013e318248dec1

8. Prain K, Woodhall M, Vincent A, et al. AQP4 Antibody Assay Sensitivity Comparison in the Era of the 2015 Diagnostic Criteria for NMOSD. Front Neurol. 2019;10:1028. Published 2019 Oct 4. 
doi:10.3389/fneur.2019.01028

9. McCracken L, Zhang J, Greene M, et al. Improving the antibody-based evaluation of autoimmune encephalitis. Neurol Neuroimmunol Neuroinflamm. 2017;4(6):e404. Published 2017 Oct 11. doi:10.1212/NXI.0000000000000404

10. Jarius S, Paul F, Aktas O, et al. MOG encephalomyelitis: international recommendations on diagnosis and antibody testing. J Neuroinflammation. 2018;15(1):134. Published 2018 May 3. doi:10.1186/s12974-018-1144-2

11. Molina RD, Conzatti LP, da Silva APB, et al. Detection of autoantibodies in central nervous system inflammatory disorders: Clinical application of cell-based assays. Mult Scler Relat Disord. 2020;38:101858. doi:10.1016/j.msard.2019.101858 